\title{
Rheological properties of aging thermosensitive suspensions
}

\author{
Eko H. Purnomo, Dirk van den Ende, ${ }^{*}$ Jorrit Mellema, and Frieder Mugele \\ Physics of Complex Fluids, Department of Science and Technology, University of Twente, P.O. Box 217, 7500 AE Enschede, Netherlands
}

(Received 1 June 2007; published 28 August 2007)

\begin{abstract}
Aging observed in soft glassy materials inherently affects the rheological properties of these systems and has been described by the soft glassy rheology (SGR) model [S. M. Fielding et al., J. Rheol. 44, 323 (2000)]. In this paper, we report the measured linear rheological behavior of thermosensitive microgel suspensions and compare it quantitatively with the predictions of the SGR model. The dynamic moduli $\left[G^{\prime}(\omega, t)\right.$ and $\left.G^{\prime \prime}(\omega, t)\right]$ obtained from oscillatory measurements are in good agreement with the model. The model also predicts quantitatively the creep compliance $J\left(t-t_{w}, t_{w}\right)$, obtained from step stress experiments, for the short time regime $\left[\left(t-t_{w}\right)<t_{w}\right]$. The relative effective temperature $\mathcal{X} / \mathcal{X}_{g}$ obtained from both the oscillatory and the step stress experiments is indeed less than $1\left(\mathcal{X} / \mathcal{X}_{g}<1\right)$ in agreement with the definition of aging. Moreover, the elasticity of the compressed particles $\left(G_{p}\right)$ increases with increased compression, i.e., the degree of hindrance and consequently also the bulk elasticity $\left(G^{\prime}\right.$ and $\left.1 / J\right)$ increases with the degree of compression.
\end{abstract}

DOI: 10.1103/PhysRevE.76.021404

PACS number(s): 83.80.Hj, 83.60.Bc, 83.80.Kn

\section{INTRODUCTION}

Pastes are not the simple materials as they appear to be. It seems that they have a memory: after a force has been applied, they recover and move back in the opposite direction, as Weitz stated in a comment [1] on an investigation of the long time mechanical behavior of highly concentrated microgel suspensions by Cloitre et al. [2]. Pastes are highly concentrated suspensions of soft particles: due to excluded volume effects the particles are deformed and possibly compressed by their neighbors. Microgel particles form a class of macromolecules intermediate between highly branched polymers and macroscopic polymer networks [3]. A microgel particle is an intramolecularly cross-linked, soluble macromolecule of colloidal dimensions. The size depends on the degree of cross linking and the nature of the solvent and is comparable to very high molecular weight polymers; its internal structure is that of a swollen network. Therefore the effective volume fraction of the microgel particles in the suspension can be controlled during the experiment by adjusting the temperature and/or solvent quality. Microgels are used as binders in organic coatings and in food products, while pastes in general are applied in various area's, such as pharmaceutical, food, and cosmetic industries.

The relaxation times of aging materials, such as the microgel particle pastes, increase as the material ages and therefore a thermodynamic equilibrium will never be achieved. Experimental evidence for aging and "close to aging" behavior stems from a wide range of soft glassy materials such as polymers [4,5], colloidal suspensions [2,6-11] foams [12], and also living cells [13-16]. The mechanical properties of aging materials depend on the age of the system just as in the case of amorphous polymers. However, the explanation given in the past for the aging of amorphous polymers does not hold for colloidal suspensions, because the energies involved in the restructuring and equilibration processes are larger than the thermal energy $k_{B} T[17,18]$.

\footnotetext{
*h.t.m.vandenEnde@utwente.nl
}

In an earlier paper [11] we have shown that a thermosensitive PNIPAM (poly- $N$-isopropylacrylamide) microgel suspension is a good model system for a colloidal glass exhibiting aging behavior. We also have shown, by exploiting their thermosensitive properties, that these suspensions can be rejuvenated not only by shearing the sample mechanically but also by a thermal quench. The viscoelastic properties of these PNIPAM suspensions can be described quantitatively quite well with the soft glassy rheology (SGR) model. In this paper, we extend this quantitative comparison to step stress experiments. We also measure the viscoelastic moduli for two other thermosensitive microgel particle systems, another PNIPAM with slightly larger particles and a PNIPAM-PNIPMAM (poly- $N$-isopropylacrylamide-poly$N$-isopropylmethacrylamide) core-shell system. Also for these systems both the linear oscillatory response and the step stress response are in quantitative agreement with the model. The relative effective temperature $\mathcal{X} / \mathcal{X}_{g}$ extracted from both types of experiments is less than 1 which indeed shows, according to the SGR model, that the suspensions are in the aging state.

The paper is organized as follows. In Sec. II we describe the experimental systems and the method and procedures used in this study. In Sec. III we report our results and explain them qualitatively. In Sec. IV we introduce the key elements of the SGR model and finally in Sec. V a quantitative comparison between the SGR model and the experimental results is discussed. From this comparison, we extract the relative effective temperature $\mathcal{X} / \mathcal{X}_{g}$ of our systems for various conditions as well as the elasticity $G_{p}$ of the compressed particles. Our conclusions are formulated in Sec. VI.

\section{EXPERIMENTAL METHOD}

\section{A. Sample synthesis}

The original PNIPAM system (hereafter called P-1) has been prepared following the procedure described in [19] using $1.18 \times 10^{-4} \mathrm{~g} / \mathrm{ml}$ of sodium dodecyl sulfate as surfactant. The sample is purified by centrifugation $(15000 \mathrm{rpm}, 2.5$ 


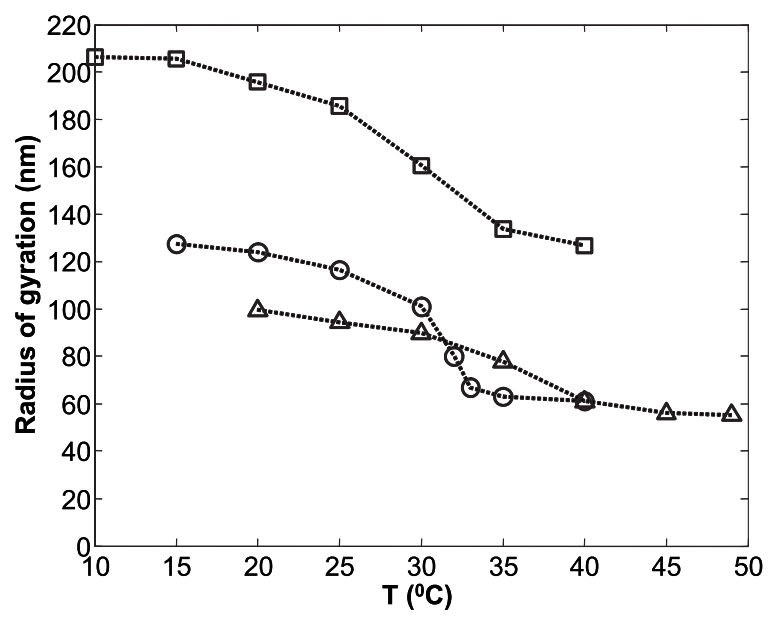

FIG. 1. The radius of gyration $R_{g}$ of the thermosensitive P-1 $(\bigcirc)$, P-2 $(\square)$, and P-P $(\triangle)$ microgel particles. The lines are a guide for the eye.

hours) and redispersed in ultrapure water at $25^{\circ} \mathrm{C}$. This procedure is repeated 4 times.

The second PNIPAM system (hereafter called P-2) was obtained from the Materials Science and Technology of Polymers group at the University of Twente. P-2 was synthesized according to the method described in [20]. After synthesis the microgel particles were dialyzed using a semipermeable membrane (molecular weight cutoff $=12$ 000-14 000) for 1 week.

The core-shell PNIPAM-PNIPMAM sample (hereafter called P-P) was obtained from the Complex Fluids group of the RWTH Aachen University. The core of these particles consists of cross-linked PNIPAM while the shell contains the PNIPMAM component. The method to synthesize these particles is described in [21]. After synthesis the sample was purified in three cycles of ultracentrifugation $(50000 \mathrm{rpm}$, 45 minutes) and redispersion in bidistilled water. The solid concentrations of these three suspensions have been determined using gravimetry.

\section{B. Sample characterization}

\section{Light scattering}

Static light scattering experiments are performed to determine the radius of gyration $R_{g}$ of the microgel particles as function of the temperature. Very dilute suspensions (mass fraction $0.0001 w / w)$ are used for these experiments. The radius of gyration is determined from the form factor $P(q)$ $=I(q) / I(0)$ where $q$ is the wave number and $I$ is the measured intensity, using a Guinier's plot, i.e., plotting $\ln (I)$ versus $-q^{2} R_{g}^{2} / 3$.

The radius of gyration of the three different soft microgel particles has been given in Fig. 1 as a function of the temperature $T$. The microgel particles are swollen at low temperatures and the radius of gyration decreases with increasing temperature. For $T>35^{\circ} \mathrm{C}$, the radius of gyration does not decrease any further for both the P-1 and the P-2 system. This indicates that the particles are fully shrunken which is

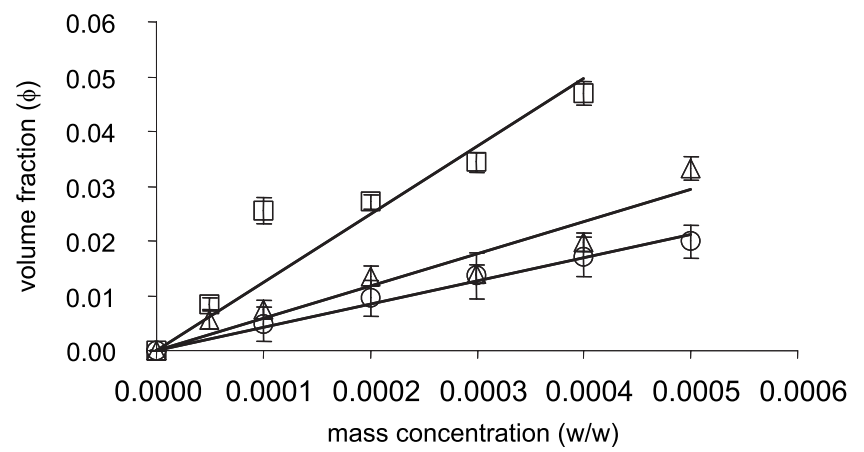

FIG. 2. The volume fraction $\phi$ as calculated from the viscosity data using the Einstein relation of dilute P-1 $(\square)$, P-2 $(\triangle)$, and P-P (○) suspensions as functions of their mass fraction $m(w / w)$. The lines indicate the linear regressions of the corresponding data.

in agreement with earlier reports $[19,20,22]$. For the coreshell P-P system, $R_{g}$ continues to decrease up to $T \sim 45^{\circ} \mathrm{C}$. This further decrease stems from the shrinking of the PNIPMAM shell which has a transition temperature of about $44{ }^{\circ} \mathrm{C}[21]$.

\section{Effective volume fraction}

The volume fraction $\phi$ of the diluted suspensions is determined using Einstein's relation, $\eta / \eta_{s}=1+2.5 \phi$ for $\phi \ll 1$. This equation describes the linear increase of the viscosity due to the increase of the volume fraction of the suspended particles. The viscosity of water $\eta_{s}$ and that of the suspensions $\eta$ are measured with a Haake RS600 using a cone and plate geometry (cone angle, $2^{\circ}$, diameter, $60 \mathrm{~mm}$ ). The viscosity of the P-1 and P-P suspensions are measured at $24{ }^{\circ} \mathrm{C}$ and the viscosity of the P-2 suspension at $20{ }^{\circ} \mathrm{C}$. At these temperatures the microgel particles are swollen and do not show any attractive interaction [23]. The shear rate used to measure the viscosity is kept below $200 \mathrm{~s}^{-1}$ to avoid secondary flow effects.

The data presented in Fig. 2 show that the volume fraction $\phi$ of a dilute suspension increases linearly with the mass fraction $m$, i.e., $\phi=a m$. The proportionality constant $a$ is determined from the slopes of the curves in Fig. 2: $a=124,59$, and 42 for the system P-1, P-2, and P-P, respectively.

For the rheological aging experiments samples of P-1, P-2, and P-P are used with mass fractions of $0.07,0.10$, and $0.07(w / w)$, respectively. According to the linear relation, found above for very low mass fractions, the corresponding volume fraction $\phi$ is about 8.7, 5.9, and 2.9, respectively. For these high mass fractions this means that the volume available for a single microgel particle in the suspension is only a fraction of its free volume at that temperature: $0.11,0.17$, and 0.34 for P-1, P-2, and P-P, respectively. So the particles are strongly compressed in these suspensions, feeling a high mutual repulsion which increases with increasing mass fraction and the effective volume fraction is $\phi_{\text {eff }} \sim 1$.

\section{Rheological aging experiments}

The rheological experiments are also performed with the Haake RS600 rheometer using the cone and plate geometry, 
(a)

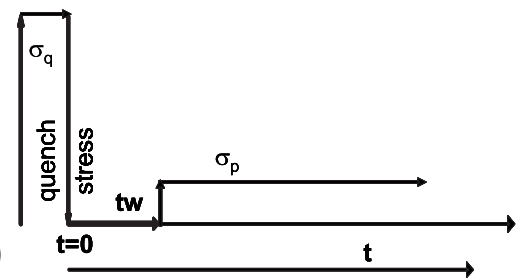

(b)

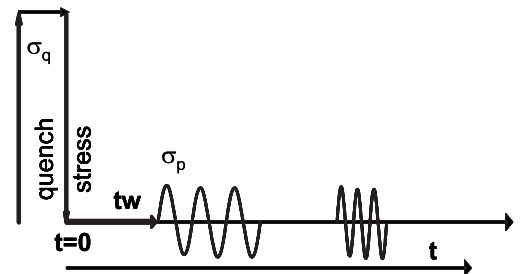

FIG. 3. (a) Schematic procedure of a step-stress and (b) an oscillatory stress measurement in the aging study.

mentioned above. The general protocol for rheological aging studies involves sample loading, quenching, waiting, and measuring the rheological properties. Sample loading is performed as follows: At $36{ }^{\circ} \mathrm{C}$ (i.e., in the shrunken state) two milliliters of concentrated suspension are injected on the lower plate of the rheometer. Subsequently, the cone is positioned at the right height and the system is cooled down to the desired temperature.

After loading and cooling the sample, it is mechanically quenched: a stress $\sigma_{q}$ well above the yield stress $\sigma_{y}$ is applied for $60 \mathrm{~s}$ to erase the memory of the sample. The end of the quench is defined as $t=0$.

Before the rheological measurements are performed, the sample is kept at rest for a certain waiting time $t_{w}$, during which no stress is applied. After this waiting time, a step or oscillatory stress $\sigma_{p} \ll \sigma_{y}$ is applied to examine the rheological properties of the sample. In the step-stress scenario [Fig. 3(a)], a constant small stress is applied and the strain response is measured. In the oscillatory measurements, an oscillating probe stress $\sigma_{p} \ll \sigma_{y}$ is applied to measure the elastic and loss modulus [Fig. 3(b)]. The results shown are an average over typically three or more independent experimental runs.

\section{EXPERIMENTAL RESULTS}

\section{A. Quench}

Since an aging material never reaches thermodynamic equilibrium, the only way to achieve a well-defined initial state is to prepare the sample far from equilibrium. One way to achieve this is by applying a stress well above the yield stress such that the sample undergoes a strong shear flow. Figure 4 shows the effect of amplitude $\sigma_{q}$ and duration $t_{q}$ of the quench on the strain recovery of the material. After the quench, the strain decreases with the elapsed time $t$ due to the elasticity of the sample. Figure 4(a) shows that for stresses $\sigma_{q}$ well above the yield stress $\sigma_{y} \sim 48 \mathrm{~Pa}$, the measured strain recovery is independent of the applied stress $\sigma_{q}$, i.e., all the curves measured at different $\sigma_{q}$ collapse on each other. However, if the quench stress $\sigma_{q}=50 \mathrm{~Pa}$ is close to the
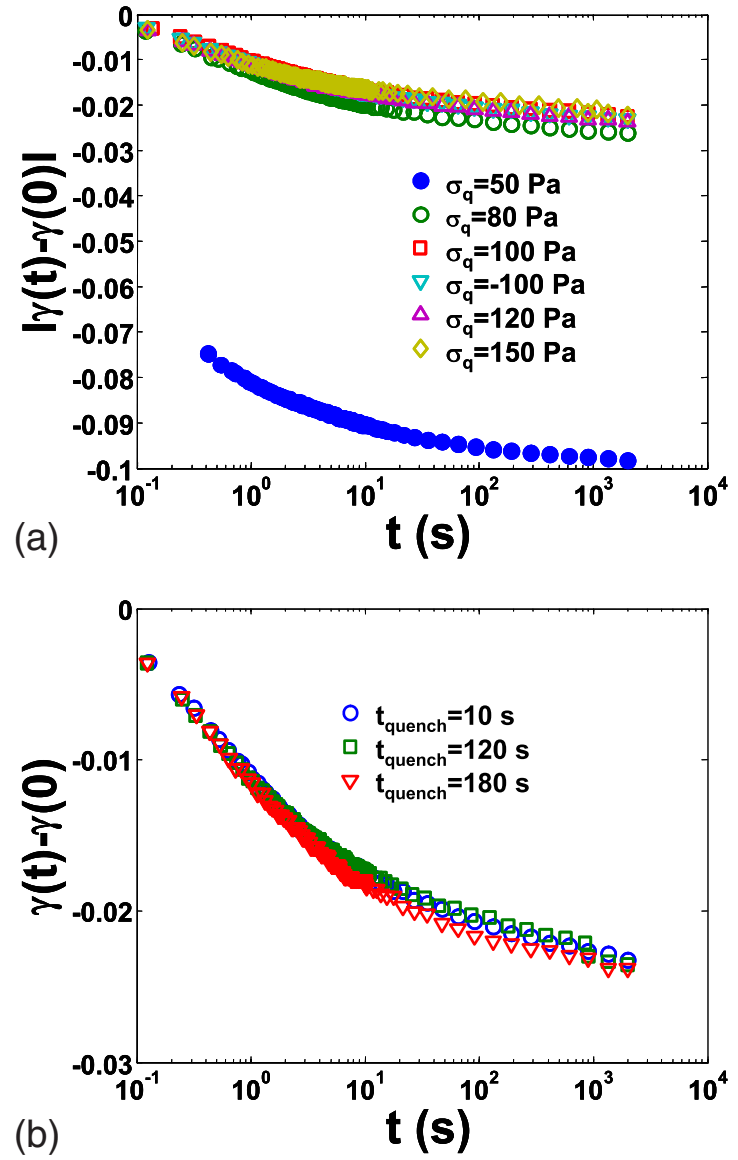

FIG. 4. (Color online) (a) The effect of quench stress $\left(t_{q}\right.$ $=60 \mathrm{~s}$ ) and (b) quench duration on the strain recovery of the P-1 suspensions after the flow cessation $\left(T=24^{\circ} \mathrm{C}\right)$.

yield stress $\sigma_{y} \sim 48 \mathrm{~Pa}$, the strain recovery is different from the other curves: because the structure is hardly destroyed by these stresses the elasticity of the sample is much larger. Figure 4(b) shows that the strain recovery is independent of the quench duration $t_{q}$ too, provided that $\sigma_{q} \gg \sigma_{y}$. In this study we use $\sigma_{q}=100 \mathrm{~Pa}$.

The collapse of the strain recovery curves obtained from the samples quenched with different stress amplitudes well above the yield stress and stress durations indicates that the system has been prepared in a well-defined initial state. On the other hand, when the stress is close to the yield stress $\left(\sigma_{q}=50 \mathrm{~Pa}\right)$, the quench is insufficient to erase the sample memory as shown by the fast elastic jump at short time in the strain recovery curve. Moreover it is also observed that the absolute strain recovery obtained from a quench with $\sigma_{q}=$ $-100 \mathrm{~Pa}$ does not differ from the recovery obtained from a $\sigma_{q}=+100 \mathrm{~Pa}$ quench. This means that the recovery process is independent of the stress direction.

\section{B. Step stress}

In a step-stress experiment, after some time $t_{w}$ measured from the cessation of the quench, a small stress, typically $\sigma_{p}=1 \mathrm{~Pa}$, is applied to the sample and the strain response is recorded. In Fig. 5(a) a typical strain response $\gamma(t)-\gamma\left(t_{w}\right)$, 

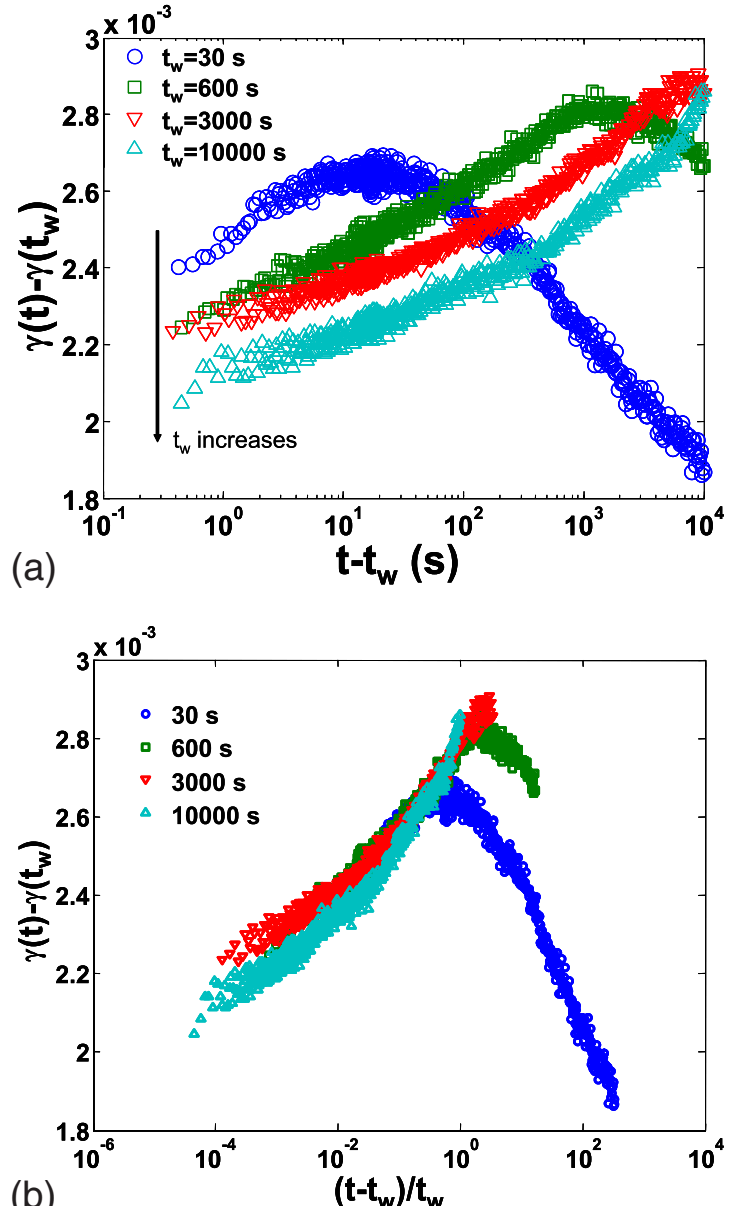

FIG. 5. (Color online) (a) The strain response of the P-1 suspension at $24{ }^{\circ} \mathrm{C}$ measured at different waiting times when the probe stress is smaller than the critical stress. (b) The same data plotted as a function of $\left(t-t_{w}\right) / t_{w}$.

where $\gamma\left(t_{w}\right)$ is the strain just before the probe stress was applied, has been plotted as a function of $t-t_{w}$ at $T=24{ }^{\circ} \mathrm{C}$ for the P-1 suspension and four different waiting times. Curves measured at other temperatures look similar.

Typically, in the first stage of the recovery process the strain increases due to the step stress applied at $t_{w}$. At a certain moment the strain reaches a maximum and the recovery direction reverses because the sample still feels the effect of the initial quench, due to its long relaxation times. For $t_{w}=30 \mathrm{~s}$, the strain peaks at $t-t_{w}=10 \mathrm{~s}$. The peak shifts to longer times $t-t_{w}$ as the waiting time increases and is hardly visible for the samples with $t_{w}=3000 \mathrm{~s}$ and $10^{4} \mathrm{~s}$. It is also observed that in the first stage, before the peak is reached, $\gamma(t)-\gamma\left(t_{w}\right)$ is lower for older samples (longer $\left.t_{w}\right)$.

The decrease of $\gamma(t)-\gamma\left(t_{w}\right)$ as the sample ages shows that the sample is more elastic at longer $t_{w}$. This behavior can be explained in terms of yielding events of the particles caged by their neighbors. The cage can be considered as an energy well. When no shear is applied the yielding is purely driven by the "effective noise temperature." Due to this yielding the elastic energy stored in the particle is dissipated. Because on the average the new cage will represent a deeper trap (less deep traps are faster depopulated) the escape rate goes down

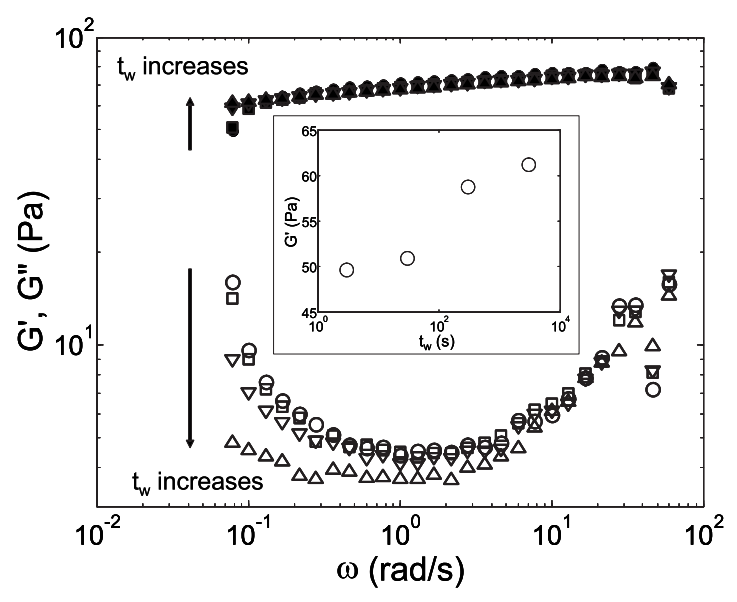

FIG. 6. The moduli $G^{\prime}$ (filled symbols) and $G^{\prime \prime}$ (open symbols) of a concentrated P-2 suspension $(0.10 w / w)$ as a function of the frequency measured at $20{ }^{\circ} \mathrm{C}$ for different waiting times: $t_{w}$ $=3 \mathrm{~s}(\bigcirc), 30 \mathrm{~s}(\square), 300 \mathrm{~s}(\nabla), 3000 \mathrm{~s}(\triangle)$. Inset: the increase of $G^{\prime}$ as a function of $t_{w}$ at $\omega=0.0628$.

and accordingly the relaxation times increase. Therefore an old sample undergoes fewer yielding events, resulting in less energy dissipation. Moreover, the tendency towards deeper traps as time evolves implies also an increase of the elasticity of the sample.

Figure 5(a) shows that $\gamma(t)-\gamma\left(t_{w}\right)$ depends not only on the elapsed time $t-t_{w}$ but also on the age of the sample $t_{w}$. Therefore, in Fig. 5(b) $\gamma(t)-\gamma\left(t_{w}\right)$ has been plotted as a function of $\left(t-t_{w}\right) / t_{w}$. Now all the curves collapse onto a single master curve for $\left(t-t_{w}\right) / t_{w}<1$. For longer times, $\left(t-t_{w}\right) / t_{w}$ $>1$, the curves do not collapse because here the initial quench dominates the recovery process so it will depend only on the elapsed time $t$ and not on the waiting time $t_{w}$.

\section{Linear viscoelasticity}

Figure 6 shows the viscoelastic moduli $G^{\prime}$ and $G^{\prime \prime}$ of the $\mathrm{P}-2$ suspension for different waiting times $t_{w}$ measured at $20^{\circ} \mathrm{C}$. The data for the P-1 and the P-P suspension look very similar; moreover the P-1 results have been presented in [11]. The elastic modulus $G^{\prime}$ increases only slightly with frequency in the interval considered. On the other hand, the loss modulus $G^{\prime \prime}$ is at least one order of magnitude smaller and passes through a minimum.

The effect of waiting time $t_{w}$ is most pronounced in the behavior of $G^{\prime \prime}$ at low frequencies $(\omega<1 \mathrm{rad} / \mathrm{s})$. The loss modulus $G^{\prime \prime}$ decreases with increasing waiting time. The effect of $t_{w}$ is also observed, but less pronounced, in $G^{\prime}$. The elastic modulus of older samples is slightly higher than that of a fresh sample (see the inset in Fig. 6). Again, the increase of $G^{\prime}$, at a fixed frequency, and the decrease of $G^{\prime \prime}$ with sample age can be explained by the growth of the relaxation times as the sample ages. The older sample with longer relaxation times undergoes fewer yielding events and therefore less energy will be dissipated, i.e., $G^{\prime \prime}$ goes down with age while the elasticity $G^{\prime}$ increases due to the increasing trap depth. 
The same line of reasoning can be used to explain the decrease of $G^{\prime \prime}$ as function of $\omega$. As $\omega$ increases, the time available for yielding during one cycle decreases and therefore less yielding events will occur. This results in a lower dissipation rate and a decrease in the loss modulus $G^{\prime \prime}$. The behavior of $G^{\prime \prime}$ at high frequencies is attributed to the local viscous effect and the Brownian motion of the particle [11]. These contributions are not due to particle-particle interactions, so they are age independent and can be represented as $G^{\prime \prime}=\omega \eta_{\infty}^{\prime}+c \omega^{0.5}$, where $\eta_{\infty}^{\prime}$ is the high frequency viscosity of the suspension and $c$ is a fitting parameter.

\section{SGR MODEL}

For a more quantitative analysis, we compare the experimental data with the SGR model [24-26]. The SGR model, based on Bouchaud's trap model, is intended to describe the rheological properties of repulsive glasses. The shared properties with soft glassy materials are metastability and structural disorder; the particles are too compressed to relax independent of each other and so, the particles are trapped by their neighboring particles. The traps can be thought to be surrounded by an energy barrier which the trapped particle must overcome before it can escape from the trap resulting in a local rearrangement of particles.

In the SGR model, the material is conceptually divided into many mesoscopic element. An element may be seen as the representation of a particle or a cluster of particles. The macroscopic strain $\gamma$ applied to a system is distributed homogeneously throughout the system and therefore the macroscopic strain rate is equal to the local strain rate $i$ experienced by an element $\dot{\gamma}=\dot{l}$.

The energy barrier $E$ of an element, or the trap depth, is equal to $k l_{y}^{2} / 2$ where $k$ is the elastic constant and $l_{y}$ is the yield strain of an element. The yielding in an unsheared or unstrained material is accompanied by the rearrangement of the neighboring particles. This type of yielding is termed noise-induced yielding and is represented in the model by a "effective temperature" $\mathcal{X}$ and proportional to $\exp (-E / \mathcal{X})$. The yielding probability increases if a macroscopic strain is applied. This type of yielding is termed strain-induced yielding and proportional to $\exp \left[-\left(E-\frac{1}{2} k l^{2}\right) / \mathcal{X}\right]$. Even though strain-induced and noise-induced yielding are discussed in different ways, the SGR model captures them both; due to the local strain $l$, the barrier to overcome is reduced to $E$ $-\frac{1}{2} k l^{2}$. Due to the disordered nature of the soft glassy material, each element will have a different yield strain. Therefore, to obtain the number of elements that yield over a certain time interval, we need to use the average yielding rate of the elements. In the model, the probability $P(E, l, t)$ that an element will be in a state with yield energy $E$ and internal strain $l$ is given by

$$
P(E, l, t)=\int_{-\infty}^{\infty} P_{q}(E, l, t, m) d m+\int_{0}^{\tau} \int_{-\infty}^{\infty} P_{r}(E, l, t, m, s) d m d s,
$$

where $P_{q}(E, l, t, m) d E d l d m$ is the distribution of elements with a yield energy between $E$ and $E+d E$, and a strain be- tween $l$ and $l+d l$, present at time $t$ which were formed in the quench with an initial strain between $m$ and $m+d m$. $P_{r}(E, l, t, m, s) d E d l d m d s$ represents the distribution of elements with a yield energy between $E$ and $E+d E$, and a strain between $l$ and $l+d l$, present at time $t$ which were formed in the time interval $(s, s+d s)$ with an initial strain between $m$ and $m+d m$. Evaluation of the integral in Eq. (1) [26] gives

$$
\begin{aligned}
P(E, l, t)= & P_{0}(E) e^{-\Omega Z(t, 0,0)} \delta[l-\gamma(t)] \\
& +\int_{0}^{t} Y(s) \rho(E) e^{-\Omega Z[t, s,-\gamma(s)]} \delta\{l-[\gamma(t)-\gamma(s)]\} d s,
\end{aligned}
$$

where $P_{0}(E)$ is the distribution of yield energies $E$ directly after the quench, $\Omega=\exp (-E / \mathcal{X})$ and $Z$ is defined as

$$
Z(t, s, u)=\int_{s}^{t} \exp \left(\frac{1}{2 x}\left[u+\gamma\left(t^{\prime}\right)\right]^{2}\right) d t^{\prime} .
$$

$Y(s)$ represents the yielding rate and

$$
\rho(E)=\frac{1}{\mathcal{X}_{g}} \exp \left(-E / \mathcal{X}_{g}\right)
$$

is the renewal distribution for the yield energies $E$, with $\mathcal{X}_{g}$ $=\langle E\rangle$, while $x=\mathcal{X} / \mathcal{X}_{g}$.

Once $P(E, l, t)$ is known, the constitutive equation (in one dimension) can be expressed as

$$
\sigma(t)=G_{p}\langle l\rangle=G_{p} \iint l P(E, l, t) d E d l
$$

which results in

$$
\sigma(t)=G_{p}\left(\gamma(t)-\int_{0}^{t} G_{\rho}(t-s) Y(s) \gamma(s) d s\right),
$$

where

$$
G_{\rho}(s)=\int_{0}^{\infty} e^{-s \Omega} \Omega^{-x} d E .
$$

Since we consider pastes that are far from equilibrium, we cannot define, strictly speaking, $G^{\prime}$ and $G^{\prime \prime}$ on the basis of a memory relaxation function. Therefore, we use a practical definition for $G^{\prime}$ and $G^{\prime \prime}$. Experimentally, $G^{\prime}$ and $G^{\prime \prime}$ are determined by correlating the measured stress response $\sigma(t)$ with the oscillatory shear $\gamma(t)=\gamma_{0} \cos (\omega t)$ over $m$ periods via

$$
\begin{aligned}
G^{\prime} & =\frac{\omega}{m \pi \gamma_{0}} \int_{t_{0}-m \pi / \omega}^{t_{0}+m \pi / \omega} \sigma(t) \cos (\omega t) d t, \\
G^{\prime \prime} & =\frac{-\omega}{m \pi \gamma_{0}} \int_{t_{0}-m \pi / \omega}^{t_{0}+m \pi / \omega} \sigma(t) \sin (\omega t) d t .
\end{aligned}
$$

Substitution of the constitutive equation, Eq. (4), in the expressions for $G^{\prime}$ and $G^{\prime \prime}$ yields

$$
G^{\prime}\left(\omega, t_{0}\right)=G_{p}-\frac{G_{p} \omega}{m \pi} \int_{t_{0}-m \pi / \omega}^{t_{0}+m \pi / \omega} M(t) \cos (\omega t) d t,
$$




$$
G^{\prime \prime}\left(\omega, t_{0}\right)=\frac{G_{p} \omega}{m \pi} \int_{t_{0}-m \pi / \omega}^{t_{0}+m \pi / \omega} M(t) \sin (\omega t) d t,
$$

where $M(t)$ is defined by

$$
M(t)=\int_{0}^{t} G_{\rho}(t-s) Y(s) \cos (\omega s) d s .
$$

The numerical integration of Eqs. (7) and (8) is not straightforward due to the functional dependence of $G(s)$ and $Y(s)$ on $s$. In the Appendix A, an efficient and accurate method is described to evaluate $G^{\prime}(\omega, t)$ and $G^{\prime \prime}(\omega, t)$. For quick comparison with the experimental data, using the exact asymptotic form of the yielding rate $[Y(t)$ $\left.=t^{x-1} / \Gamma(x) \Gamma(x) \Gamma(1-x)\right]$ the following asymptotic relation as given by [26] can be used:

$$
G^{*}(\omega, t)=G_{p}\left(1-\frac{1}{\Gamma(x)}(i \omega t)^{x-1}\right) \text { for } x<1,
$$

where $\Gamma$ is the well-known $\Gamma$ function.

The same SGR model can be applied to a step-stress experiment. However, since the overall strain recovery due to the quench is not described in the model, only the extra contribution due to the small probe stress is predicted. For a step-stress experiment, the stress as a function of time is given by $\sigma(t)=\sigma_{0} H\left(t-t_{w}\right)$ and the strain response can be written as $\gamma=\sigma_{0} J\left(t-t_{w}, t_{w} ; \sigma_{0}\right)$. Dividing Eq. (4) by the applied stress $\sigma(t)$ results in

$$
1 / G_{p}=J\left(t-t_{w}, t_{w}\right)-\int_{t_{w}}^{t} J\left(s-t_{w}, t_{w}\right) Y(s) G_{\rho}(t-s) d s .
$$

Solving this integral equation will result in an expression for $J\left(t-t_{w}, t_{w}\right)$. For comparison with the experimental results, however, again a more simple asymptotic relation can be used [26],

$$
J\left(t-t_{w}, t_{w}\right)=\frac{1+c\left[\left(t-t_{w}\right) / t_{w}\right]^{1-x}}{G_{p}},
$$

for

$$
\frac{\left(t-t_{w}\right)}{t_{w}} \ll 1,
$$

where $c$ is a constant.

\section{EXPERIMENT VERSUS MODEL}

It has been shown in Fig. 6 that the viscoelastic moduli $G^{\prime}$ and $G^{\prime \prime}$ of the P-2 suspension depend on both the frequency $\omega$ and the age $t_{w}$. Due to the experimental protocol used, in which we apply a frequency sweep from low to high frequency, the total age $t$ of the sample includes not only the waiting time $t_{w}$ but also the time required to perform the oscillation at its frequency and the preceding frequencies. In Fig. 7, we show $G^{\prime}$ and $G^{\prime \prime}$ of the P-2 that are plotted as a function of $\omega t$. The $G^{\prime}$ and $G^{\prime \prime}$ measured at different ages

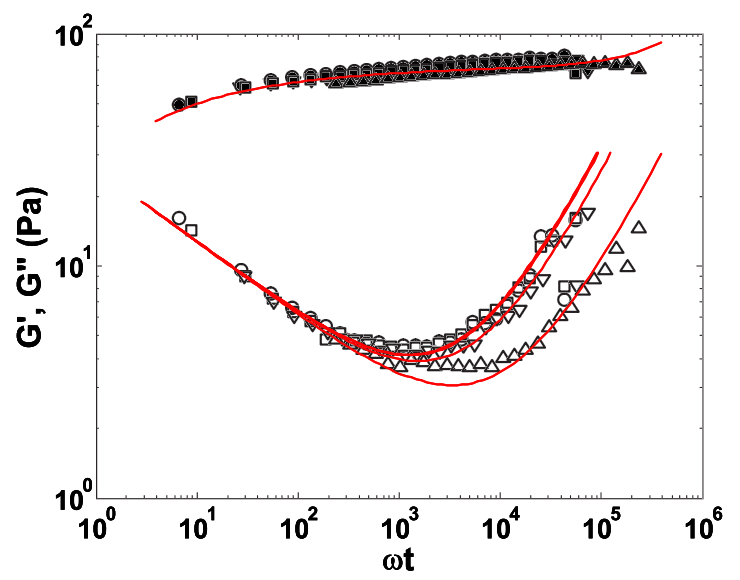

FIG. 7. (Color online) $G^{\prime}(\omega, t)$ and $G^{\prime \prime}(\omega, t)$ plotted as a function of $\omega t$ for the concentrated P-2 suspension $(0.10 w / w)$. Different symbols correspond to different experimental waiting times, as in Fig. 6. Solid lines show model calculations, see text for details.

collapse onto a single master curve especially at $\omega t<10^{3}$ where the particle-particle interactions dominate the behavior of the moduli. The deviation of $G^{\prime \prime}$ from the master curve is again due to the contribution from the local viscous effect and the Brownian motion.

The viscoelastic moduli of the aging P-2 suspension is then compared quantitatively to the prediction of the SGR model presented as lines in Fig. 7. The viscoelastic moduli can be calculated either from Eqs. (7) and (8) or Eq. (10). In addition to the $G^{\prime}$ and $G^{\prime \prime}$ calculated from the SGR model, we also include the Brownian motion $G_{D}^{\prime}(\omega)=G_{D}^{\prime \prime}(\omega)$ $=c \omega^{1 / 2}$ and the local viscosity contribution $G_{S}^{\prime \prime}(\omega)=\eta_{\infty}^{\prime} \omega$ to account for the behavior at high frequency. The high frequency viscosity $\eta_{\infty}^{\prime}=0.2 \mathrm{~Pa}$ is independently measured and then used in the calculation.

The relative effective temperature $x=\mathcal{X} / \mathcal{X}_{g}$ and the elasticity of the compressed particles $G_{p}$ obtained from this comparison are 0.68 and $80 \mathrm{~Pa}$, respectively. The relative effective temperature which is smaller than unity means that the sample is indeed in the aging state. In this state, the sample never achieves an equilibrium because the average relaxation time $\left[\tau(t)=\Gamma(x) \Gamma(x) \Gamma(1-x) / t^{x-1}\right]$ grows with its age.

The quantitative comparison of the predictions of the SGR model to the step-stress data is presented in Fig. 8. The data is presented as a creep compliance, $J=[\gamma(t)$ $\left.-\gamma\left(t_{w}\right)\right] / \sigma_{p}$. The comparison is made only for $\left(t-t_{w}\right) / t_{w} \ll 1$ because the SGR model only predicts the evolution of the strain caused by the applied stress and neglect the strain recovery originating from the quench step. The model assumes that the sample is strain free after the stress removal in the quench stress, which is not the case in our experiments. Due to the elasticity of the sample, the remaining strain exist as the sample relaxes back as observed in the decrease of the strain at $\left(t-t_{w}\right) / t_{w}>1$. Incorporating the strain recovery originating from the quench step to the model is expected to describe the creep compliance not only for $\left(t-t_{w}\right) / t_{w} \ll 1$ but also for $\left(t-t_{w}\right) / t_{w} \gg 1$. However, this is beyond the scope of this paper.

The model presented as solid lines in Fig. 8 can be calculated numerically using Eq. (12). Two parameters, $x=\mathcal{X} / \mathcal{X}_{g}$ 

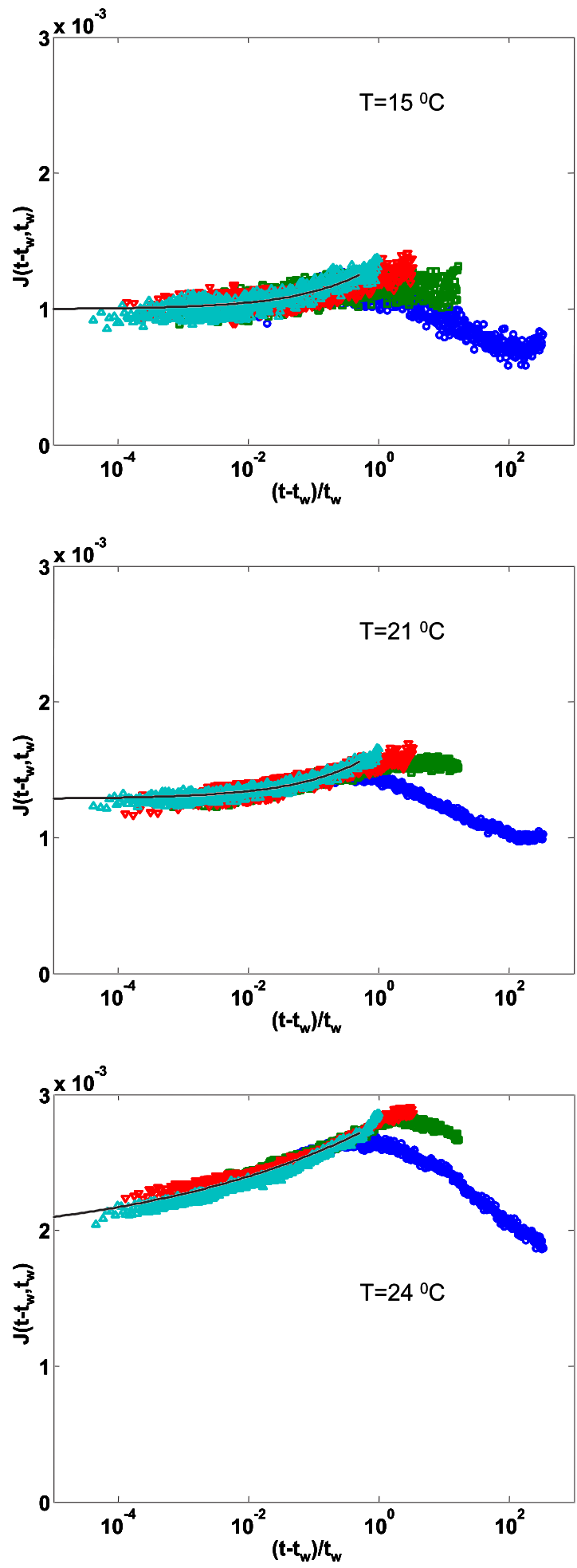

FIG. 8. (Color online) $J\left(t-t_{w}, t_{w}\right)$ as a function of $\left(t-t_{w}\right) / t_{w}$ for the P-1 suspension at different ages $\left(30 \mathrm{~s}-10^{4} \mathrm{~s}\right)$ and measured at different temperatures. The solid lines represent the predictions of the SGR model.

and $G_{p}$, are fitted in this comparison. The relative noise temperatures, $x=0.58 \pm 0.05,0.60 \pm 0.03$, and $0.88 \pm 0.01$ as obtained for $T=15^{\circ} \mathrm{C}, 21{ }^{\circ} \mathrm{C}$, and $24{ }^{\circ} \mathrm{C}$, respectively, show that the suspensions are in the aging regime. The $x$ value of

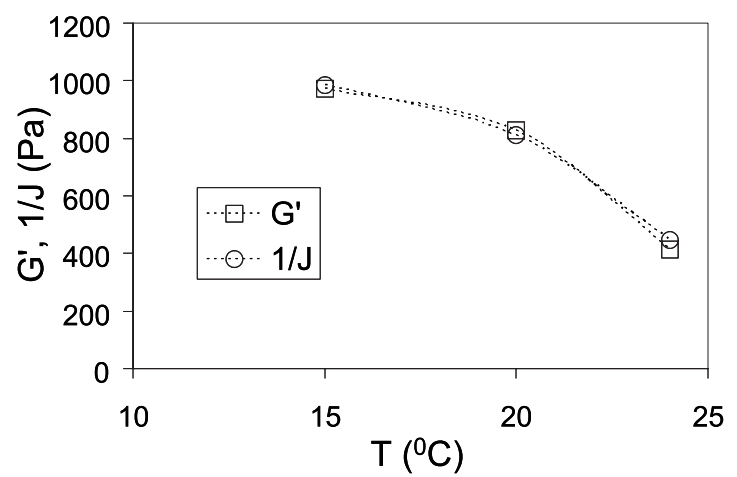

FIG. 9. The elasticity, $G^{\prime}$ and $1 / J$, of the P-1 suspension for $t_{w}=600 \mathrm{~s}$ at different temperatures.

P-1 suspension at $24{ }^{\circ} \mathrm{C}$ obtained from the step-stress method is higher than the one obtained from the oscillatory method $(x \approx 0.62[11])$. We speculate that the difference stems from the fact that the probe stress applied in one direction (step stress) partially rejuvenates the sample and increases the $x$ value.

The elasticity of the compressed particles increases with decreasing temperature, $G_{p}=1002 \pm 11,778 \pm 5$, and $536 \pm 16 \mathrm{~Pa}$ for $T=15^{\circ} \mathrm{C}, 21^{\circ} \mathrm{C}$ and $24^{\circ} \mathrm{C}$, respectively. The result is comparable to the compressed particle elasticity $G_{p}$ obtained from the dynamic measurements [11]. The elasticity of the particle increases as the particles are more compressed due to the increase of $R_{g}$ while the macroscopic volume is preserved.

Figure 8 also shows that the sample at lower temperature has smaller $J\left(t-t_{w}, t_{w}\right)$ which means that the sample is more elastic. In Fig. 9 we plot the elasticity of the P-1 suspension measured both with oscillatory $G^{\prime}[11]$ and step-stress experiments $1 / J$ at $t_{w}=600 \mathrm{~s}$. The data for different waiting times look similar. The higher elasticity of the sample at lower temperature is related to the increase of the elasticity of its constituents which are the individual particles. The increase of the particles elasticity collectively increases the bulk elasticity. This increase in the bulk elasticity can also be described in term of the yielding process. At lower temperature the particles are more constrained and therefore there is less yielding which is responsible for the energy dissipation. Because the energy dissipation is small, the elasticity of the sample increases.

\section{CONCLUSIONS}

In summary, we found that both $G^{\prime}(\omega)$ and $G^{\prime \prime}(\omega)$ as well as the strain response in step-stress experiments of highly concentrated soft microgel particles depend strongly on their age. The aging behavior is quantitatively described by the soft glassy rheology model. It is in agreement with the model predictions for systems in the aging state, a relative effective temperature less than unity $\left(\mathcal{X} / \mathcal{X}_{g}<1\right)$ is found consistently for both types of measurements. Also with respect to the elasticity, we find a consistent behavior: the elasticity of the individual particles $G_{p}$ is found to increase with decreasing temperature in accordance with decreasing bulk elasticity- 
for which we find $G^{\prime} \simeq 1 / J$ even though the system is not in equilibrium.

Overall, our measurements confirm that the SGR model correctly captures the aging behavior of microgel suspensions. The discrepancies seen in the long-term strainrelaxation behavior show that the stress- and strain-free initial conditions assumed in the SGR model are not fulfilled in the typical mechanical quench protocol experiments. While this problem can probably be overcome by both improved quench protocols or extensions of the model, the deeper question of the origin of the effective noise temperature and its absolute value still remain to be solved. We expect that nonlinear rheological experiments currently in progress in our laboratory will provide information on both the absolute average energy barrier (and thus the noise temperature) and the evolution of the characteristic relaxation time $[\tau(t)]$ of the aging suspensions.

\section{ACKNOWLEDGMENTS}

The authors thank O. Tagit from the Materials Science and Technology of Polymers group at the University of Twente, W. Richtering and M. Keerl from the Complex Fluids group of the RWTH Aachen University as well as M. H. G. Duits and J. L. Lopulissa, for assistance with the sample preparation and the light scattering experiments. This work has been supported by the Foundation for Fundamental Research on Matter (FOM), which is financially supported by the Netherlands Organization for Scientific Research (NWO).

\section{APPENDIX}

Since both $G_{\rho}(s)$ and $Y(s)$ decay quickly with $s$ but have also a long time tail, accurate integration of Eqs. (7) and (8) must be done with care. The integration interval $[0-t]$ is split up in subdomains to probe the product $G_{\rho}(s) Y(t-s)$ properly: $[0,10],[10,100], \ldots,\left[10^{m}, t-10^{m}\right], \ldots,[t-100, t$ $-10],[t-10, t]$ where $m$ was chosen such that $10^{m}<t / 2$ $<10^{m+1}$. Moreover, partial integration is used to handle the cosine and sine contributions correctly also if the time step becomes of the order of one period or even larger,

$$
\int_{t_{n-1}}^{t_{n}} f(s) \cos (\omega s) d s=\left(\frac{1}{\omega^{2}} f^{\prime}(s) \cos (\omega s)+\frac{1}{\omega} f(s) \sin (\omega s)\right)_{t_{n-1}}^{t_{n}},
$$

$$
\int_{t_{n-1}}^{t_{n}} f(s) \sin (\omega s) d s=\left(\frac{1}{\omega^{2}} f^{\prime}(s) \sin (\omega s)-\frac{1}{\omega} f(s) \cos (\omega s)\right)_{t_{n-1}}^{t_{n}} .
$$

In these expressions, it has been assumed that the derivative of $f(s)=G_{\rho}(s) Y(t-s)$ is almost constant over the interval $\left[t_{n-1}, t_{n}\right], f^{\prime}(s)=\left[f\left(t_{n}\right)-f\left(t_{n-1}\right)\right] /\left(t_{n}-t_{n-1}\right)$. Each subinterval was again divided into 100 time steps over which Eqs. (A1) and (A2) were evaluated.
[1] D. A. Weitz, Nature (London) 410, 32 (2001).

[2] M. Cloitre, R. Borrega, and L. Leibler, Phys. Rev. Lett. 85, 4819 (2000).

[3] H. Senff and W. Richtering, Colloid Polym. Sci. 278, 830 (2000).

[4] L. C. E. Struik, Ann. N.Y. Acad. Sci. 279, 78 (1976).

[5] H. Montes, V. Viasnoff, S. Jurine, and F. Lequeux, J. Stat. Mech.: Theory Exp. 3 ( 2006) 71.

[6] R. E. Courtland and E. R. Weeks, J. Phys.: Condens. Matter 15, S359 (2003).

[7] C. Derec, A. Ajdari, G. Ducouret, and F. Lequeux, C. R. Acad. Sci., Ser. IV Phys. 1, 1115 (2000).

[8] C. Derec, G. Ducouret, A. Ajdari, and F. Lequeux, Phys. Rev. E 67, 061403 (2003).

[9] V. Viasnoff and F. Lequeux, Phys. Rev. Lett. 89, 065701 (2002).

[10] D. Bonn, P. Coussot, H. T. Huynh, F. Bertrand, and G. Debrégeas, Europhys. Lett. 59, 786 (2002).

[11] E. H. Purnomo, D. van den Ende, J. Mellema, and F. Mugele, Europhys. Lett. 76, 74 (2006).

[12] S. Cohen-Addad, H. Hoballah, and R. Höhler, Phys. Rev. E 57, 6897 (1998).

[13] B. Fabry, G. N. Maksym, J. P. Butler, M. Glogauer, D. Navajas, N. A. Taback, E. J. Millet, and J. J. Fredberg, Phys. Rev. E
68, 041914 (2003).

[14] P. Bursac, G. Lenormand, B. Fabry, M. Oliver, D. A. Weitz, V. Viasnoff, J. P. Butler, and J. J. Fredberg, Nat. Mater. 4, 557 (2005).

[15] R. E. Laudadio, E. J. Millet, B. Fabry, S. S. An, J. P. Butler, and J. J. Fredberg, Am. J. Physiol.: Cell Physiol. 289, C1388 (2005).

[16] L. Deng, X. Trepat, J. P. Butler, E. Millet, K. G. Morgan, D. A. Weitz, and J. J. Fredberg, Nat. Mater. 5, 636 (2006).

[17] B. Abou and F. Gallet, Phys. Rev. Lett. 93, 160603 (2004).

[18] P. Wang, C. Song, and H. A. Makse, Nat. Phys. 2, 526 (2006).

[19] J. Gao and Z. Hu, Langmuir 18, 1360 (2002).

[20] R. H. Pelton and P. Chibante, Colloids Surf. 20, 247 (1986).

[21] I. Berndt, J. S. Pedersen, P. Lindner, and W. Richtering, Langmuir 22, 459 (2006).

[22] M. Stieger, W. Richtering, J. S. Pedersen, and P. Lindner, J. Chem. Phys. 120, 6197 (2004).

[23] M. Stieger, J. S. Pedersen, P. Lindner, and W. Richtering, Langmuir 20, 7283 (2004).

[24] P. Sollich, F. Lequeux, P. Hébraud, and M. E. Cates, Phys. Rev. Lett. 78, 2020 (1997).

[25] P. Sollich, Phys. Rev. E 58, 738 (1998).

[26] S. M. Fielding, P. Sollich, and M. E. Cates, J. Rheol. 44, 323 (2000). 